\title{
Article \\ What Group Theory Can Do for You: From Magmas to Abstract Thinking in School Mathematics
}

\author{
Joaquin Marc Veith ${ }^{1, *(1)}$ and Philipp Bitzenbauer ${ }^{2}$ (I) \\ 1 Institut für Mathematik und Angewandte Informatik, Stiftung Universität Hildesheim, \\ 31141 Hildesheim, Germany \\ 2 Physikalisches Institut, Friedrich-Alexander-Universität Erlangen-Nürnberg, 91058 Erlangen, Germany; \\ philipp.bitzenbauer@fau.de \\ * Correspondence: veith@imai.uni-hildesheim.de
}

Citation: Veith, J.M.; Bitzenbauer, P. What Group Theory Can Do for You: From Magmas to Abstract Thinking in School Mathematics. Mathematics 2022, 10, 703. https://doi.org/ $10.3390 /$ math10050703

Academic Editor: Jay Jahangiri

Received: 18 January 2022

Accepted: 22 February 2022

Published: 23 February 2022

Publisher's Note: MDPI stays neutral with regard to jurisdictional claims in published maps and institutional affiliations.

Copyright: (C) 2022 by the authors. Licensee MDPI, Basel, Switzerland This article is an open access article distributed under the terms and conditions of the Creative Commons Attribution (CC BY) license (https:// creativecommons.org/licenses/by/ $4.0 /)$.

\begin{abstract}
In this paper, we present a novel concept for an abstract algebra course at the secondary school level. Against the backdrop of findings from mathematics education research, we discuss benefits that arise from teaching abstract algebra to secondary school students. We derive suitable content items as well as educational ideas on how to implement them into mathematics classrooms. We build on frameworks from mathematics education research to design a teaching-learning sequence that enables a beneficial learning trajectory across three units- the result is a coherent teaching concept that encompasses Magmas in a hands-on way. It can be used for either extra-curricular activities or advanced courses in mathematics classes, and can be of benefit for mathematics learners.
\end{abstract}

Keywords: algebra; groups; magmas; secondary school; mathematics education

\section{Introduction}

Over the past two decades, numerous studies have shown how abstract algebra is connected to school mathematics and how studying the concepts of abstract algebra influences one's view on mathematics in general. For example, with a course for mathematics teachers, Wasserman [1] has shown that presenting K-12 teachers with the ideas of abstract algebra not only increased their content knowledge but also influenced their beliefs and even their classroom practices. In other words, it vastly transformed their way of thinking about mathematics. The participants of the course argued that learning about group properties made them realize underlying concepts of school mathematics (specifically in arithmetic and algebra), putting them into perspective, which in turn helped them paint a bigger picture. This specific reasoning was also touched upon by McCrory et al.'s [2] in-depth discussion of knowledge for algebra teaching. In another study by Even [3] multiple teachers in the context of an in-service training voiced the opinion that dealing with these concepts advanced their understanding about what mathematics is and what doing mathematics actually means. On the other hand, approaching mathematics disregarding structures can lead to misconceptions, as has been shown for different mathematics topics (cf. [4]). So not only does abstract algebra provide learning opportunities for the nature of mathematics itself, it also improves knowledge about specific subdisciplines (cf. [5]). In addition to arithmetic and algebra, for exmaple, the study of group theory marks great value for the context of geometric symmetries in school mathematics [6,7].

These connections were studied even further by Wasserman [8], looking at all possible content areas across elementary, middle and secondary mathematics that may be influenced by knowledge about concepts of abstract algebra. Exploring abstract algebra yet again through the lens of school mathematics, this work constructed a progression of four content areas (Solving Equations, Structure of Sets, Inverses and Arithmetic Properties) that are potentially transformed by the perspective abstract algebra provides. 
However, simply because teachers view their field of education differently does not mean these changes also translate to the students or help students understand school algebra better in any way. In fact, multiple empirical studies have shown that this is not the case, the constructs teacher knowledge in abstract algebra and student achievement in school algebra do not seem to correlate significantly (cf. [3,9-16]). This raises the question whether the mentioned benefits that come with abstract algebra are used to its full potential when leaving students out of the equation. In addition, since the ultimate goal of mathematics education is always enhancing students' perceptions and understanding of mathematical concepts, these observations result in one pressing question: Can these abstract algebra courses be tweaked so that students themselves can profit from them? And if so, do they profit in a similar way?

This question is by no means a new one. It was the driving force behind the educational reform 'new math' which overhauled the entire mathematical curricular infrastructure during the 1960s and 1970s. It was eventually dismissed, but during the time a lot of educational work has been produced on the topic and it is been laying waste for the better part of a century now. This unsatisfying coexistence of the numerous possibilities of abstract algebra (better understanding of arithmetic properties, discovering underlying concepts of equations, positive impact on structural knowledge, better understanding of concatenations) and the lack of ambition to make use of it led us to design a new teaching concept for abstract algebra at secondary school levels which in the following we will introduce.

At first, we will present a brief historical overview of the 'new math' reform as well as present the key ideas and notions that have been produced by numerous didacts at that time before comparing them to modern research on this topic. One may interpose that these ideas are partially over 50 years old and thus outdated, but the mathematics has remained the same and it is the first and only attempt ever of bringing abstract algebra down to school level at a large scale. Additionally, since empirical studies on these ideas are also dated and new educational ideas have been produced over the years, they can be merged and studied thoroughly with modern methods, potentially yielding new conclusions. Therefore, the guidelines for our proposal will not only take modern approaches into account but we will also pick up ideas of the past and try to synthesize both bodies of work to a homogeneous concept.

\section{The New Math Era: A Brief Historical Overview}

The purpose of this section is to provide unfamiliar readers a quick perspective on the new math movement to better contextualize the referenced literature in the next sections. A full historical in-depth look can be found in [17].

On 4 October 1957, the Soviet Union succeeded in launching a satellite into Earth orbit for the first time. This historic achievement and clear demonstration of technical progress triggered numerous impulses that ultimately penetrated the education system of the US and many European countries. In response to what is now called the "Sputnik shock", several attempts were made to raise the level of education, specifically in scientific related subjects, in order to remain internationally competitive. In his article "The New Math and Its Aftermath", David Rappaport [18] writes: "Sputnik was also responsible for the development of the new math programs in the elementary schools and in the high schools. That the Soviet Union could be ahead of the United States in some area of technology came as shock to most Americans. The result was a demand for drastic change in the mathematics and science curricula in the school programs. The National Science Foundations, as well as a number of private foundations, made available millions of dollars to schools and to individuals do create new mathematics programs" (p. 563).

This gave rise to the SMG (School Mathematics Study Group) as a major project aimed at revising the traditional curriculum. Numerous writers produced books and learning materials for mathematics teaching; a new mathematics was emerging under the name "New Math". In 1959, two years after the Sputnik Shock, the OEEC (today OECD) convened 
a two-week seminar in Royaumont, France. At the center of the discussions held there is one topic alone: new thinking in mathematics education; the topics of this seminar are publicly documented in [19], key figures are Hans-Georg Steiner from Germany and Jean Dieudonné from France. The entire subject of mathematics is to be restructured and reoriented. New ways of thinking are to be introduced, and the structures behind mathematical concepts are to be brought to light. In concrete terms, this means, among other things: structural mathematics (today called abstract algebra), which has been relegated to university, must be integrated into the school curriculum.

Since our teaching concept will be studied in Germany we will specifically highlight the movements development in Germany: In 1966, about 7 years after the Royaumont Seminar, the project manifests and the reform points discussed in the seminar get published in volume form under the title "Synopsis for Modern School Mathematics" [20]. The educational reform is made binding nationwide, teacher training and curricular changes are implemented and school literature is revised. However, with teachers and parents being unable to cope with the sudden changes, the reform quickly receives a bad image in public and its purpose is heavily discussed and critiqued (cf. [21]). This ultimately leads to the reform being dismissed in the late 1970s, ending its short-lived existence.

Many mathematics educators at the time, however, argued that the abolition of the reform was as hastily as its implementation and many studies claimed that students who engaged in abstract algebra performed better in school algebra tests than their peers (cf. [22]). A brief description of the movements' development in the US including a comprehensive overlook of all the covered topics of abstract within the new math reform can be found in [22].

\section{Historical and Contemporary Ideas on Teaching Abstract Algebra}

In this section, we present and discuss didactical ideas and research on the topic of teaching abstract algebra. We will highlight both the work of researchers from the 'new math' era as well as contemporary work. The goal is to identify learners' difficulties with topics of abstract algebra, how to avoid them and what contents of abstract algebra are suited when dealing with this subject for the first time.

\subsection{Educational Key Findings of the 'New Math' Era}

Mathematicians and mathematics educators have been very productive during the 'new math' era. So productive in fact, that presenting all the developed ideas will be an impossible task in the context of this contribution. We therefore decided to present two recurring themes of the literature that seemed to be the least controversial and thus provide the most common ground.

At first, however, one has to note that bringing abstract algebra all the way down to school mathematics is without any doubt an ambitious goal. It requires careful consideration of contents and compelling educational ideas in order to be fruitful to the student. These requirements can be categorized in a broad manner, resulting in basic landmarks when introducing abstract algebra to pupils. In this regard, Nöbauer [23] enunciated three fundamental rules:

1. Algebraic structures must not be introduced in a formal way or independent of tangible models. The axiomatic nature can be discussed if an opportunity presents itself, but it is of secondary importance.

2. Mathematical terms may only be introduced if they can be demonstrated or illustrated on the basis of already existing terms.

3. The goal of abstraction is only possible if the underlying concepts have been studied thoroughly.

To understand the third rule, one has to keep in mind that originally algebraic structures were planned to be introduced in the lower level of secondary schools. For our concept, however, only the upper level is targeted, so familiarity with arithmetics and geometry can be assumed. 
The first and second rule can be explained by looking at the mathematics of the time: The axiomatic approach to mathematics which was developed by the Bourbaki group a few years prior to the 'new math' era developed a fundamentally new view on mathematics in general. Introducing mathematical structure was a new way of unifying concepts, but it required a more general thinking. Demanding from school students who are just learning about the basics of mathematics to form this perspective was highly controversial. If at all, it was seen to be possible if the abstract concepts of structures appeared explicitly, meaning that they are made visible and physically engaging via concrete models. This is, by far, the most recurring condition among the entire body of research during the 1960s.

\subsubsection{No Axiomatic Group Theory}

As mentioned above and in the first fundamental rule by Nöbauer, axiomatic group theory was neither a goal nor a desideratum. In an important paper Freudenthal [7] argued that when exploring groups one should not start by defining it in an abstract way and then look at examples (basically the way every mathematical content is introduced in university). Instead it should happen vice versa: The educational process should move from the particular to the general. However, Dubinsky et al. [24] noted that it still might be difficult for students to abstract concepts they've seen in specific and concrete examples. After all, the group concept is not a single abstract idea, but rather a collection of multiple abstract ideas.

It is worth mentioning that not all mathematics educators voiced their opinions against an axiomatic treatment. For example, Liermann [25] argued that the concept of groups is ideal for a first look at the axiomatic nature of mathematics, since it is less convoluted compared to other school mathematics areas such as geometry or set theory. To provide another example, Kropp [26] also took a decisive stance in favor of treating axioms in class. He reasoned that the demands on the ability to abstract are only high when compared to the usual requirements in conventional contents of school mathematics or other subjects. He further argued that the transition towards abstraction is just a matter of acclimatisation and that the more one gets used to the notions the less abstract they appear.

Since the empirical research that was used to back up those statements in most cases would not suffice modern research standards, we do not dare to derive a clear "yes" or "no" for the axiomatic approach from the literature. For reasons mentioned in the next paragraph, we decided to take the less abstract approach and leave the axioms of group theory out of the concept for now. For more in-depth discussions of the time as to how the axiomatic method can or should be treated in class, we refer the reader to the articles [27-32].

\subsubsection{Magmas Instead of Groups}

As mentioned in the second and third rule, the abstract concepts have to be made very explicit by demonstrating their manifestations visually and making them engaging. Steiner, a key figure in the 'new math' era and participant of the Royaumont Seminar, had the idea in this regard to not approach abstract algebra by looking at groups right away, but looking at magmas first. He reasoned that set theory is deeply rooted in every discipline of mathematics, even in school mathematics, i.e., sets of points in the euclidean plane or space, number sets in arithmetic, domains and ranges of functions or solution sets of equations. So even though their applications may vary, the mathematical object itself is a recurring theme, suggesting that students have a better understanding of it than of any other aspect of groups [33]. Binary operations, on the other hand, are neither known nor made explicit at any point. For instance, multiplication and addition are operations solely applied to real numbers and their structural similarity as maps $\mathbb{R} \times \mathbb{R} \rightarrow \mathbb{R}$ is never discussed. Therefore, before even thinking about tackling groups, it is vital that students have a sufficiently abstract idea of binary operations [34]. For our concept, we thus deduce that while still working with groups it might be better to just view them as magmas first and embezzle additional properties. To support this, Steiner [34] derived two main principles for establishing algebraic structures: 
1. How many models in the direct mathematical periphery tap the algebraic structure? In other words, which structures draw the most from visual demonstrations?

2. Which of those algebraic structures in 1. allow for an adequate (in relation to school mathematics), deductive approach as well as an exploratory approach?

Very similar main principles were also developed by Dieudonné (cf. [35]). From these principles one can derive groups that satisfy the conditions and are, thus, suited for a first introduction. For example, Griesel [27] derived the following list:

- Dihedral groups but also the groups of symmetries of rectangles, rhombuses, rhomboids, kites, etc.

- $\quad$ Frieze groups

- Cyclic groups

- $\quad$ The additive groups of $\mathbb{Z}, \mathbb{Q}$ and $\mathbb{R}$

- $\quad$ The multiplicative groups of $\mathbb{Q}_{>0},\left\{2^{n}: n \in \mathbb{Z}\right\}$, etc.

- The orthogonal groups

This list was further refined by Leppig [36] to $D_{3}, D_{4}$ and $\mathbb{Z}_{n}$. In this article, cyclic groups are defined as groups that are generated by a single element, and, hence, can be written as

$$
G=\langle g\rangle=\left\{g, g^{2}, g^{3}, \ldots\right\}
$$

for some $g \in G$. Furthermore, the Dihedral group $D_{n}$ of order $2 n$ is defined as the subgroup of the Symetric group $S_{n}$ generated by

$$
\sigma=\left(\begin{array}{ccccc}
1 & 2 & \ldots & n-1 & n \\
2 & 3 & \ldots & n & 1
\end{array}\right) \quad \text { and } \quad \tau=\left(\begin{array}{ccccc}
1 & 2 & 3 & \ldots & n \\
1 & n & n-1 & \ldots & 2
\end{array}\right)
$$

where $\sigma$ can be interpreted as a rotation and $\tau$ can be interpreted as a reflection of the regular $n$-gon. For the refined list Leppig reasons as follows: On the one hand, congruent mappings are already part of the geometry curriculum in secondary schools, so transformations of equilateral triangles and squares can be studied without having to establish new mathematics while at the same time allowing for a visually engaging entry to group theory, making the dihedral groups $D_{3}$ and $D_{4}$ perfect candidates. On the other hand, the direct mathematical periphery are number sets, so groups like $(\mathbb{Z},+),(\mathbb{Q},+)$ or $(\mathbb{R} \backslash\{0\}, \cdot)$ are obvious candidates. It can be argued, however, that not much from those groups can be learned and instead one should try to make use of the ubiquity of arithmetic by looking at finite cyclic groups $\left(\mathbb{Z}_{n},+\right)$. Those groups have more compelling applications and are more abstract, so understanding them allows for a better understanding of the concepts of group theory. For additional justification, we will see in Section 3.2 that the learning processes of precisely those groups have been well studied and documented in recent research, so we can pick up modern ideas on how to approach those structures.

For dihedral groups the exploratory approach mentioned in the second main principle will not pose too much trouble. For cyclic groups however, some work needs to be done in order to enable students to explore them. We will present our ideas on how to explore both kinds of groups in a compelling way in Section 5.

\subsubsection{Interim Conclusions}

Summarizing the educational ideas above, from literature at the time we categorized three educational key findings (cf. Table 1).

Table 1. Key findings derived from historic research on abstract algebra teaching.

\begin{tabular}{ll}
\hline Key Finding & References \\
\hline No axiomatic group theory & {$[7,24,29]$} \\
\hline Magmas instead of groups & {$[33-35]$} \\
\hline Best groups for introducution are $D_{3}, D_{4}$ and $\mathbb{Z}_{n}$ & {$[27,36,37]$} \\
\hline
\end{tabular}


Very importantly, this already gives hints which groups are suited the most for an introductory course as well as the fact that the binary operation is of such a relevance that focusing on Magmas first is a crucial part of entering abstract algebra. This is also important with regards to practicability of the teaching concept; exploring groups would require additional activities for inverses, the neutral element and associativity. Additional activities however require more time and thus more teaching units. On the other hand, one can study properties of groups without actually naming them and generalize ideas. In order to get to abstraction, it is necessary to use the ideas - but not necessarily the language - of inverses, the neutral element and associativity [19].

\subsection{Educational Key Findings in Contemporary Research}

Modern research on the topic, as mentioned in the introduction, involved solely prospective teachers. In addition, university students differ vastly from school students, both in terms of previous knowledge and in terms of interests and beliefs. The participants in the subsequently mentioned studies obviously had a richer math education to build upon, and also are arguably somewhat drawn towards mathematics, having made the decision to teach this subject in school. On the other hand, this allows for research to dig deeper and explore more content with the participants, so a lot of insight on learning all the numerous concepts of abstract algebra is gained. Lots of study has been made on learning Groups, Subgroups, Cosets, Cayley Tables, Isomorphisms, Polynomials, Rings and Fields. For example, a collection of the scientific output can be found in the edited volume "Connecting Abstract Algebra to Secondary Mathematics, for Secondary Mathematics Teachers" [38]. Although the researchers on the field touch on many different topics, studying the literature suggests that there are three distinctive key findings for learning abstract algebra which we will present in the following.

\subsubsection{Linear Equations and Familiar Number Sets}

Students have shown to prefer working with familiar number systems when presented with objects of abstract algebra $[39,40]$. This result is not surprising since the most obvious connection between abstract algebra and school algebra lies within the fact that the number sets $\mathbb{Z}, \mathbb{Q}$ and $\mathbb{R}$ are among the first groups one can study and they are also very present in school mathematics - with the difference that in school they are just viewed as sets without any further structure. Computations that arise from binary operations in these sets (i.e., adding, subtracting, multiplying, etc.) are familiar to the students and don't require much abstraction.

Wasserman [1] and Shamash [5] have already impressively demonstrated how abstract algebra can be introduced through solving equations. At last, all of the axioms of groups and rings can be traced back to the basic needs of solving linear equations, for example:

$$
\begin{array}{ccc}
2+x & = & 3 \\
(-2)+(2+x) & = & (-2)+3 \\
((-2)+2)+x & = & 1 \\
0+x & = & 1 \\
x & = & 1
\end{array}
$$

$$
\begin{gathered}
\text { (concatenate with inverse) } \\
\text { (associativity) } \\
\text { (property of inverse) } \\
\text { (property of neutral element) }
\end{gathered}
$$

$\begin{array}{ccc}r \circ x & =s \\ r^{2} \circ(r \circ x) & =r^{2} \circ s \\ \left(r^{2} \circ r\right) \circ x & =r^{2} s \\ \operatorname{id} \circ x & =r^{2} s \\ x & =r^{2} s\end{array}$

Thus solving linear equations works exactly the same in every group and parallels can be demonstrated elegantly, as shown above where the left-hand side can be viewed in $(\mathbb{Z},+)$ (or $\mathbb{Q}$ or $\mathbb{R})$ and the right-hand side can be viewed in $\left(D_{3}, 0\right)$ (or any of its isomorphic copies). When presenting students with new knowledge it seems apparent that the proven affinity for familiar number sets should be used so it will be part of our teaching concept.

\subsubsection{Geometrical Aspects of Group Theory}

Picking up the idea of showing students concepts they are familiar with, studies have shown that such connections between school algebra and abstract algebra can easily be overlooked unless they're made very explicit $[41,42]$. One has to keep in mind that 
the general mathematical approach of unifying concepts and categorizing mathematical objects in terms of their structure is an idea that's completely absent in school mathematics. Alternatively to solving equations, these underlying connections can be revealed via geometrical groups. In a recent study by Suominen [43] where mathematicians and mathematics educators where questioned about the connections of abstract algebra and secondary school algebra, over half of the participants brought up links between abstract algebra and secondary school geometry. This not only underpins the statements about abstract algebra connecting seemingly disjoint topics within mathematics, it also shows how important it is to include geometry when teaching abstract algebra. A full list of all the connections can be found in the mentioned paper.

Additionally, according to Burn [6], permutations and symmetries are the fundamental concepts of group theory. Consequently, while being deeply connected to group theory, geometrical approaches allow for another opportunity to make connections between secondary school mathematics and abstract algebra. We conclude that studying groups that consist of geometrical transformations such as the dihedral groups $D_{3}$ or $D_{4}$ offer a lot of potential for a fruitful teaching concept.

On a side note, Burn further reports on a course on abstract algebra successfully introducing groups in a pre-axiomatic manner via geometry. This suggests yet again that the abstract axioms of group theory are not necessarily needed in order to work with groups. Given that students in secondary school level have never faced any axiomatic structure before it seems obvious that an approach without axioms will be much more accessible as the threshold will be much lower. This is recurring aspect we will come back to.

\subsubsection{Properties of Binary Operations}

In school mathematics, properties of binary operations are often taken for granted. For example, it is never questioned why addition of real numbers is commutative, and the existence of zero is obvious. However, the idea of the identity transformation of an equilateral triangle being an actual element of the set can be confusing; after all, nothing happened to the triangle [1]. So when trying to generalize such concepts, one has to evoke a sense of caution in the student. A study by Melhuish [44] has shown that students overgeneralize and conflate properties such as associativity and commutativity. In addition, they found that conceptual understanding of groups is tied to the understanding of binary operations. Especially since geometric groups will be part of the concept and most of them are non-abelian, it will be crucial to look at the properties in detail and compare them with the other groups.

Also the inverse element poses many hurdles and can't be neglected. Students have trouble seeing the conceptual familiarity of an inverse function $f^{-1}$ and a multiplicative inverse $a^{-1}$ as Wasserman [45] found using task-based interviews. The concept maps produced in the context of his study generally showed that the participants had trouble grasping the concept of inverses. In particular, it seemed that there were no differences spotted between right and left inverses. However, one can easily construct examples where such a differentiation is necessary. There is a fundamental difference between the equations $\cos ^{-1}(\cos (\pi))=\pi$ and $\cos \left(\cos ^{-1}(\pi)\right)=\pi$. The latter requiring complex numbers to be made sense of. Also, the generic square root function

$$
f: \mathbb{R}_{\geq 0} \rightarrow \mathbb{R}_{\geq 0}, x \mapsto \sqrt{x}
$$

ist not a left inverse of the square function

$$
g: \mathbb{R} \rightarrow \mathbb{R}_{\geq 0}, x \mapsto x^{2}
$$

for example $(f \circ g)(-1)=1$. The study suggests that the inverse can't properly be understood by just looking at functions since the properties of the identity element and commutativity in general seem to be disregarded or overlooked too easily in that case. So 
for dealing with inverses it is suggested to use both function sets and number sets, which is deeply connected to the first two key findings we presented.

Additionally, a large scale assessment by Brown et al. [46] has shown that an experimental approach to abstract algebra is fruitful for learning about the concepts of binary operations and groups. For our teaching concept we conclude that a hands-on approach is required for dealing with the problems that properties of binary operations seem to entail. Experimenting and physically engaging with the mathematical objects is suggested to invoke a deeper understanding of the contents.

\subsubsection{Interim Conclusions}

Considering all the problems that have been excavated in contemporary literature, we categorized 3 key findings for our teaching concept which later will justify the contents. Not only does the research deliver crucial hints as to which challenges come with teaching abstract algebra, the key findings also present solutions and ways of teaching it. To reference the key findings, we established an abbreviation for each one (cf. Table 2):

Table 2. Key findings derived from modern research on abstract algebra teaching.

\begin{tabular}{lll}
\hline Key Finding & Abbreviation & References \\
\hline $\begin{array}{l}\text { Solving Equations, } \\
\text { working with familiar number sets }(\mathbb{N}, \mathbb{Z}, \mathbb{Q}, \mathbb{R})\end{array}$ & $\mathrm{K} 1$ & {$[1,5,39,40]$} \\
\hline $\begin{array}{l}\text { Symmetries, } \\
\text { experimenting, engaging physically }\end{array}$ & $\mathrm{K} 2$ & {$[1,6,24,40,42,46,47]$} \\
\hline Properties of binary operations & $\mathrm{K} 3$ & {$[1,44,45]$} \\
\hline
\end{tabular}

From the key findings in Table 2 one can also immediately derive content areas of abstract algebra and assign them to each finding (cf. Table 3).

Table 3. Key findings derived from modern research on abstract algebra teaching as well as the contents assigned to each finding.

\begin{tabular}{ll}
\hline Key Finding & Suggested Contents \\
\hline K1 & Modular Arithmetic, Cyclic Groups \\
\hline K2 & Dihedral Groups \\
\hline K3 & Comparing $(\mathbb{R},-),(\mathbb{R},+)$ etc. \\
\hline
\end{tabular}

It is noteworthy that the contents assigned to $\mathrm{K} 1-\mathrm{K} 3$ are precisely the ones presented in Table 1. Moreover, the contents are very much in line with a list obtained by Wasserman [8] where he explored abstract algebra yet again, only this time through the lens of school mathematics, constructing a progression of four content areas that are potentially transformed by the perspective abstract algebra provides: Solving equations, structure of sets, inverses and arithmetic properties. We observe that there is a striking consensus throughout both bodies of work when it comes to selecting groups for an introductory course.

\section{Design Principles and Structure of a New Teaching Concept}

In this section, we want to take the contents previously distilled from literature and arrange them in a certain order so that the resulting learning trajectory is the most fruitful according to research. A very famous statement in this regard comes from the 'new math' critique Why Johnny Can't Add by Kline [21] and we take it as a general guideline for our concept:

"Abstraction is not the first stage, but the last stage, in a mathematical development." [p. 98] 


\subsection{Realistic Mathematical Education (RME)}

For purposes of bridging the gap between secondary school mathematics and advanced mathematics it is necessary to reverse the order in which mathematics is usually presented [48]: We will first look at examples to draw on previously existing knowledge, refining the concepts further and only in a last step present formal and abstract notions. For our teaching concept we therefore adopt the instructional design principles of RME (Realistic Mathematical Education, cf. [49]), meaning that the axiomatic and abstract nature is not the starting point but the last goal of the teaching concept. The basic idea of RME is that the construction of mathematical contents should be realistic, representing the process of constructing a definition rather than just presenting the final result. In other words, one should enter a mathematical domain with contexts that students can make sense of and only later then generalize the underlying concepts, giving students time to gradually develop an understanding. This method is especially suited for complex topics such as abstract algebra [50], hence Freudenthal [7] coined three hierarchical levels of activity when engaging with abstract algebra:

- Level 1: An algebraic property emerges implicitly in the student's intuitive activity with an example structure.

- Level 2: The property appears explicitly in the student's general description of his or her activity with the structure.

- Level 3: The student repurposes the property as a lens to classify other structures.

We intend on realizing this hierarchical process exactly as it is described above within a time frame of three teaching units. Considering students have no prior knowledge of the formal foundations this surely marks an ambitious goal that can only be achieved by trimming back the axiomatic nature of the subject at hand. The main goal throughout the entire process remains "from the concrete to the abstract". Thus, it is recommended, as described in Section 3, to start with visually accessible contents like geometrical groups. Exploring the dihedral groups $D_{3}$ and $D_{4}(\mathrm{~K} 2$ and $\mathrm{K} 3$ ) will therefore be the contents of lecture 1 and lecture 2 as they offer a great potential for visual stimuli, while exploring the more abstract modular arithmetic via cyclic groups $\mathbb{Z}_{n}(\mathrm{~K} 1)$ and unifying all the concepts will be the goal of the final lecture 3 .

\subsection{The EDUS-System}

On an additional note, for justifying the didactical approach of our teaching concept, we will use the EDUS-System. The EDUS-System is a framework designed by Lee [48], specifically tailored for guiding the transition between school algebra to abstract algebra. The acronym EDUS encompasses 4 characteristics that are central to developing a structural perspective in mathematics:

- Extending (CE) the context in which a set of existing understandings are situated. For our concept, this is incorporated by considering geometrical transformations of the equilateral triangle and the square.

- Deepening (CD) the level of existing understandings of a certain, single mathematical object. For our concept, this is incorporated by deepening the understanding of the object map and inverse map.

- Unifying (CU) existing understandings that were previously unrelated by the student under a specific overarching mathematical object. For our concept, this is incorporated by unifying the understandings of number sets and the properties of binary operations defined on them.

- Strengthening (CS) the links between existing understandings of more than one mathematical object. For our concept, this is incorporated by looking at similarities of the different sets and deriving the magma structure from those observations.

Merging the educational ideas of RME and EDUS now allows for a highly customized teaching concept of which the schedule is presented in Figure 1. 


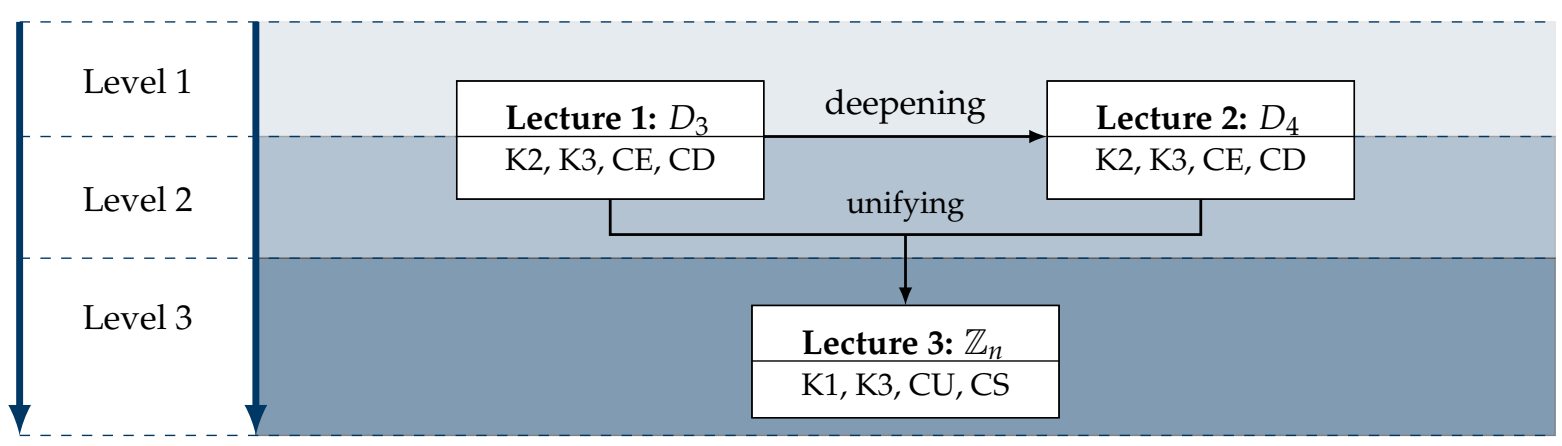

Figure 1. Schedule for the teaching concept, including the RME level, the EDUS characteristics as well as the key findings and contents for each lecture.

As illustrated in Figure 1, lectures 1 and 2 will be located within both level 1 and level 2 of RME. Looking at the non-abelian nature of these groups it will be clarified how concatenation leads to non-commutativity for the given geometrical transformations so this property of groups will first be implicit and later explicit. The identity element and inverses of the groups will be explicit only in lecture 3 when all the contents can be looked at simultaneously to reach unification. Only then we enter level 3 by comparing the new structures to the ones students have already seen in school mathematics.

Also we have decided to make K3 part of every lecture since literature suggests that problems and difficulties of binary operations seem to be the most crucial aspect about learning group theory [44].

\subsection{Connecting Abstract Algebra to Algebraic Thinking}

Since teaching abstract algebra is at its' core a teaching of the fundamentals underlying school algebra it seems apparent that another priority of this teaching concept should be the development of algebraic thinking. Although the notion of algebraic thinking in the literature is mainly tied to the learning of primary school students and thus a concept for the early stages of school mathematics, we argue that when dealing with abstract algebra the same ideas and principles apply: For example, composing functions instead of numbers is a sufficiently new step of generalization to pose new hurdles for learners, as seen in [45].

Even though the term Algebraic thinking is not a defined construct itself, much work has been done to describe the aspects of mathematics it entails. For example, according to [51], algebraic thinking encompasses among other things

- generalizing mathematical structures (cf. CE, K1, K2, K3)

- $\quad$ exploring and symbolizing functional relationships (cf. CU, K2)

- building mathematical arguments that reflect more generalized forms (cf. CE, K3)

- $\quad$ reasoning about abstract quantities (cf. CS, K1)

and according to [52] it develops from

- $\quad$ generalized arithmetic (cf. CE, K1, K3)

- functional thinking (cf. CU, K2)

- application of generalizations as a modeling language (cf. CS, K1, K3).

Here, in brackets we have assigned the characteristics according to the EDUS-System (cf. Section 4.2) and our key findings (cf. Table 2) to each item in the two lists shown above. This highlights the striking similarities between the introduced frameworks for abstract algebra and the aspects of algebraic thinking. For example, dealing with modular arithmetic builds on generalizing mathematical structures of secondary school arithmetic such as $(\mathbb{Z},+)$ or $(\mathbb{Q} \backslash\{0\}, \cdot)$. Or, as another example, modeling the transformations of different polygons can be seen as direct applications of mathematics as a modeling language. Thus, the teaching concept directly draws on the fundamental aspects that promote algebraic thinking.

Another aspect often linked to algebraic thinking is that mathematical generalization starts by identifying mathematical patterns and relationships [53] since they contribute 
to the development of process-relational skills such as mathematical representation and abstract thinking $[54,55]$. When dealing with transformations of polygons, for example, mathematical patterns can be visually identified and recurring themes can be observed (i.e., for odd $n$ the reflection lines of $D_{n}$ pass through each vertex exactly once). Additionally, since the subgroup of rotations of $D_{n}$ is always isomorphic to $\mathbb{Z}_{n}$, functoinal relationships between the different structures can be explored in an engaging way. We will elaborate these synergies in more detail in the next section, when introducing the learning trajectories of each lecture.

\section{The Hildesheim Teaching Concept of Abstract Algebra}

The concept is intended as an introductory course to abstract algebra for upper level students of secondary school (grade 11/12, 16-18 years), focusing on exploring the concept of magmas. A minimal time frame of three $90 \mathrm{~min}$. lessons is required to implement it in mathematics classrooms. In the following, we will outline our ideas in terms of didactical approaches to the contents we derived from literature in the previous sections. A full teaching manual as well as all the work sheets can be obtained from the corresponding authors in either English or German. The learning trajectory derived from Sections 3 and 4 looks as follows (cf. Figure 2):

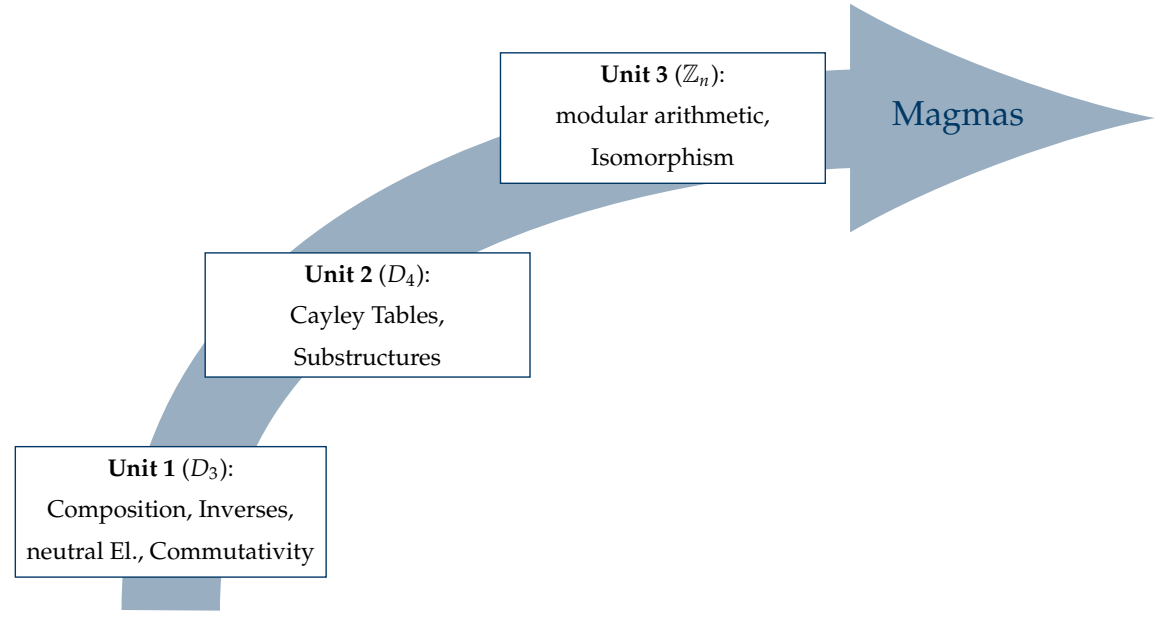

School Mathematics

Figure 2. The learning trajectory of the Hildesheim Teaching Concept of Abstract Algebra with its' contents spanning three teaching units.

The core of the concept will be exploring groups via symmetries according to Burn [6] and starting with looking at equilateral triangles as starting activities, synthesizing the ideas of Wasserman [1] and Larsen [47], but building upon them and making the concepts experienceable hands-on, keeping Dubinsky et al.'s [24] concerns in mind, and thus reducing the level of abstraction. The 'hands-on' part will be carried out by providing students with triangles made of acrylic glass, enabling physical engagement with the mathematical contents. For reasons we will later elaborate, it is important that the material is transparent. The students thus have the opportunity to perform abstract group actions in a non-abstract way by rotating and flipping their triangle. This method of students actively engaging with the important concepts supports an experiential basis for understanding ideas of abstract algebra [40].

\subsection{Unit 1: Introducing the Dihedral Group $D_{3}$}

\subsubsection{Exploring the Transformations}

In the beginning of the first unit, the students will explore all possibilities of how an equilateral triangle can be mapped onto itself. To make this process more engaging, we 
prepared acrylic glass triangles so the students can perform the geometrical transformations hands-on (cf. Figure 3).
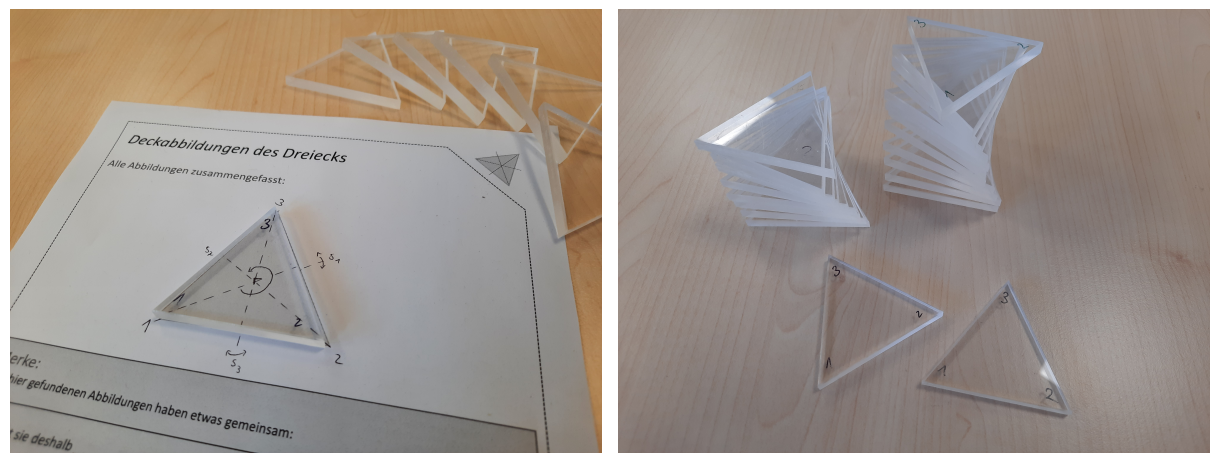

Figure 3. The acrylic glass triangles can be used to perform the elements of $D_{3}$ hands-on (own photograph). The triangles are sized exactly like the image containing all the elements on the worksheet (cf. Figure 4).

Once the students have gathered some of the possibilities the question raises whether those are all possibilities and if not, how to find the remaining ones. We introduce the first step of abstraction via numbering all the vertices of the triangle with the numbers $1-3$. The material for the triangles was chosen to be transparent so that the numbers can be seen when performing reflections, flipping the triangle on its' backside.

It can now be observed that each mapping corresponds to a certain manipulation of the vertices, and vice versa, each manipulation of the vertices corresponds to a mapping. This turns the quest of finding all the mappings into a trivial one-one simply has to find all combinations of numbers. For the first vertex, there are 3 possible numbers, for the second vertex only 2 and then the number of the last vertex is fixed. This yields $3 !=6$ possible mappings. Of course, this method will not be successful when exploring the group $D_{4}$ in the second unit, because $D_{4} \nsucceq S_{4}$ unlike $D_{3} \cong S_{3}$. But by then the students will have worked with $D_{3}$ already and have a better understanding of the contents.

Inevitably, the question will arise as to why "doing nothing" is also a legal choice of a mapping-since the motivation of having an identity element in the set is not yet feasible, we will simply describe the rotation by $360^{\circ}$ as identity mapping in id notation.

\subsubsection{Describing the Transformations}

Once all the mappings have been found the students' next activity will be describing them geometrically, assigning symbols to them and in a last step gathering all the descriptions in a tabular (cf. Table 4). The students' notes in Larsen's [47] study on students exploring the dihedral groups suggest that students will have non-intuitive ways to describe the elements of $D_{3}$, making operations and operation tables very difficult to read. This is no surprise, since they don't know yet what content comes next and what the established abbreviations will be used for. For our concept it is therefore important to denote each element of $D_{3}$ with a very visual and self-explanatory symbol. For example, the permutation (123) can be described as rotating the triangle counter-clockwise by $120^{\circ}$, so the symbol $r_{120}$ suggests itself. Analogically one has $r_{240}$ and $r_{360}$. One might interject here that only two symbols are required for describing all the elements of $D_{3}$, since it is generated by just two elements. But working with the symbol $r^{2}$ presumes knowledge of the agreed notation $r^{2}=r \circ r$, and compositions have not been introduced yet, so the symbol of each element has to be independent of the other symbols. For this reason, we will not use the notations $D_{3}=\left\{r, r^{2}, s, r s, r^{2} s\right.$, id $\}$ used in mathematics literature. 
Table 4. Two exemplary rows of the tabular containing all the elements of $D_{3}$.

Mapping

For the identity element of $D_{3}$, one can argue that since the triangle remains identical under $r_{360}$, another plausible symbol might be id. When describing the permutations (12), (23) and (13) however, we face a new problem: Since a triangle is part of the plane, the mappings are geometrically viewed as reflections. A reflection, however, can't be carried out with physical objects such as our triangles. In fact, the only way to perform the corresponding manipulations of the vertices is to rotate the triangle around a bisecting line, which requires three dimensions. Thus it has to be made very clear that the physical triangle is just a representation, and in the euclidean plane the mapping has to be described as a reflection. We finally present the students with an image containing all the mappings (cf. Figure 4), the triangle in the image is of the same size as the glass triangles, so one can put the triangle on the paper and perform the transformations as shown by the arrows in the image.

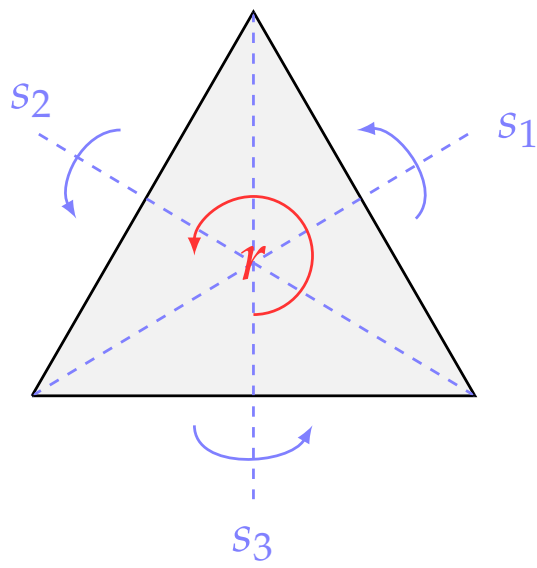

Figure 4. Image containing the elements of $D_{3}$.

Before moving on to the next step of the teaching unit, we gather all the mappings and put them in a set: $D_{3}=\left\{s_{1}, s_{2}, s_{3}, r_{120}, r_{240}\right.$, id $\}$.

\subsubsection{Exploring the Composition}

Exploring the composition of the transformations in $D_{3}$ poses two central problems:

1. The (for students) somewhat unintuitive definition of composition, and 
2. compositions involving reflections.

Firstly, a composition of two maps $f: A \rightarrow B$ and $g: B \rightarrow C$ is defined by $(g \circ f)(a)=$ $g(f(a))$ for all $a \in A$. This means, even though $g$ comes first when reading $g \circ f$, it comes last in the mathematical sense, because arguments of maps are usually denoted on the righthand side. One could write images as $(a) f$ and then proceed with $(a)(f \circ g)=((a) f) g$, so the order of execution is coherent with the order of reading, but over the years the notion (a) $f$ has more or less completely vanished. This dispute has to be addressed before any compositions in $D_{3}$ can be computed.

Secondly, compositions with reflections can be very misleading. Since the reflection lines in the descriptions are tied to the corresponding vertex number, it seems obvious that the reflection lines also get transformed. For example, when computing $s_{1} \circ r_{120}$ one may assume that the rotation also rotates the bisecting line, effectively turning $s_{1}$ into $s_{2}$ if it is performed afterwards (cf. Figure 5).

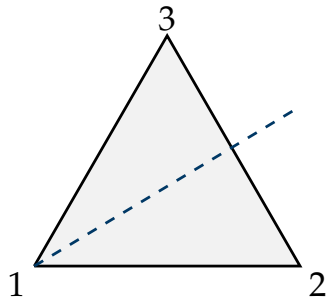

2

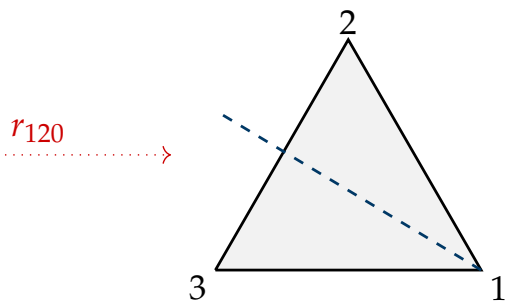

$S_{1}$

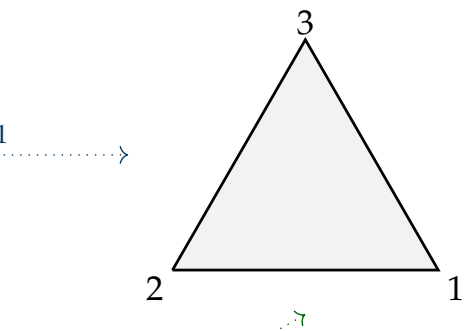

$S_{3}$

Figure 5. When also rotating the object used to describe $s_{1}$, the reflection can change under certain compositions, leading to fundamental errors. Obviously $s_{1} \circ r_{120} \neq s_{3}$.

It is therefore crucial to note that the reflection lines never change positions, i.e., if the left vertex is assigned the number 1 yielding the name $s_{1}$ for the corresponding reflection, the reflection line will always go through the left vertex (cf. Figure 6). To avoid this error we instruct the students to put their triangles on the sheet with the image containing all the elements (cf. Figure 3)-this way the reflection lines stay in position when rotating the triangles.

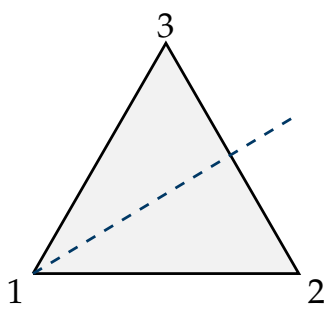

$r_{120}$



$S_{1}$

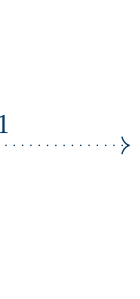

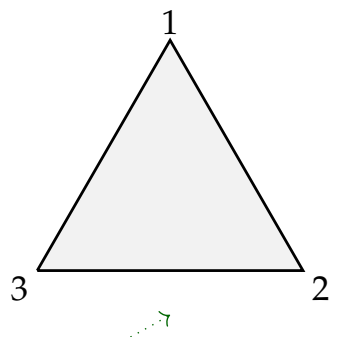

$S_{2}$

Figure 6. Keeping in mind that the reflection lines don't change yields the correct result $s_{1} \circ r_{120}=s_{2}$.

Another crucial part when working with compositions for the first time is noting that in general geometrical transformations do not commute. In our concept we immediately 
address this fact by letting students compute both $r_{120} \circ s_{1}$ and $s_{1} \circ r_{120}$ and compare the results.

The last crucial part when exploring compositions is noting that when composing any two elements of $D_{3}$ it will always result in another element of $D_{3}$, meaning that the set is closed under composition. One can verify this explicitly by computing all possible 36 combinations and filling of a Cayley Table. In our concept however, this will happen in the second teaching unit. For now, one can justify this fact with a compelling argument: If every element of $D_{3}$ maps the triangle to itself, then composing any two of those elements will also map the triangle to itself-since there are only 6 such possible mappings and we identified all of them, the result must again be an element of $D_{3}$.

\subsubsection{Exploring the Group Structure of $D_{3}$}

In the last section of the first teaching unit, we will explore some aspects of the group structure of $D_{3}$ for purposes of later generalizing our findings in the last unit. In this regard, we can explore two more aspects with the students: Inverses and the neutral element.

When computing compositions of the form id $\circ r_{120}, s_{1} \circ \mathrm{id}$, etc. it will quickly become obvious that id has no impact on the composition. Leaving the triangle identical always means that the outcome of the composition is solely determined by the other component. The identity element is unique in this regard, and we can state that for any $x \in D_{3}$ we have $x \circ \mathrm{id}=\mathrm{id} \circ x=x$. With having established the neutral element, we can move on and observe that each transformation of the triangle can be reversed. Exploring the reverse transformations with the students and gathering them in a table will yield Table 5 .

Table 5. The elements of $D_{3}$ and their inverses.

\begin{tabular}{ccccccc}
\hline Transformation & $s_{1}$ & $s_{2}$ & $s_{3}$ & $r_{120}$ & $r_{240}$ & id \\
\hline $\begin{array}{c}\text { Reverse } \\
\text { Transformation }\end{array}$ & $s_{1}$ & $s_{2}$ & $s_{3}$ & $r_{240}$ & $r_{120}$ & id \\
\hline
\end{tabular}

This can be further formalized by saying that for each element $x \in D_{3}$ there is an element $x^{-1} \in D_{3}$ reversing it, meaning that $x \circ x^{-1}=\mathrm{id}$ and $x^{-1} \circ x=\mathrm{id}$. By looking at the table it is also clear that the reverse transformations are unique for each element. It is expectable for students to argue that the inverse of $r_{120}$ is $r_{-120}$. By letting them perform the transformation $r_{-120}$ and looking at the vertices of the resulting triangle, it will be easy to identify $r_{-120}=r_{240}$.

To conclude the teaching unit one can deepen the concepts by computing more advanced compositions, such as $\left(s_{2} \circ r_{240}\right)^{-1}$ or finding all $x \in D_{3}$ that solve the equation $s_{1} \circ x=r_{120}$. For the latter mentioned exercise, the students may either

- $\quad$ try out all six possible $x \in D_{3}$ by drawing the transformations as seen in Figure 6,

- $\quad$ try out all six possible $x \in D_{3}$ physically by taking the glass triangle or

- $\quad$ note that one can reverse the reflection $s_{1}$ and thus compute $x=s_{1}^{-1} \circ r_{120}$.

If time allows it, in a last activity the students can explore that each element of $D_{3}$ can be expressed by using only $r_{120}$ and $s_{1}$, leading to the conclusion that all the transformations of the triangle are built out just of two transformations, so in a sense $r_{120}$ and $s_{1}$ can be viewed as the "atoms" of triangle transformations.

\subsection{Unit 2: Introducing the Dihedral Group $D_{4}$}

In the second of the three teaching units, we will first recap the learnings of the first unit and then guild upon them by exploring the group $D_{4}$. The students are by now familiar with the concept behind the geometrical transformations dihedral groups consist of. This means that the contents of composition, inverses, the neutral element and commutativity do not require an equally in-depth discussion anymore. Many of the educational ideas 
elucidated in Section 5.1 can be implemented in the same way so we will not go into detail anymore with regards to those.

\subsubsection{Cayley Tables}

The first activity in the second unit will consist of establishing the Cayley Table for $D_{3}$, this both acts as a recap so the relevant concepts are present within the students minds and as a setup for looking at substructures at a later point of this unit. As computing all possible combinations might be time consuming and end up being a dull repetitive exercise, we shortcut this by presenting a Cayley Table that's already halfway filled (cf. Table 6).

Table 6. Cayley Table for $D_{3}$.

\begin{tabular}{ccccccc}
\hline$\circ$ & id & $r_{120}$ & $r_{240}$ & $s_{1}$ & $s_{2}$ & $s_{3}$ \\
id & id & & $r_{240}$ & $s_{1}$ & & $s_{3}$ \\
$r_{120}$ & $r_{120}$ & $r_{240}$ & id & $s_{3}$ & $s_{3}$ \\
$r_{240}$ & & id & & id & & \\
$s_{1}$ & $s_{1}$ & $s_{2}$ & & & id \\
$s_{2}$ & $s_{2}$ & & & $r_{120}$ & & id \\
$s_{3}$ & $s_{3}$ & & $s_{2}$ & & \\
\hline
\end{tabular}

When filling the table we of course have to explain how this is done. One has to decide for either of two possibilities: rows first or columns first. For our concept we decided to go row first and then column, meaning that, for example, in the row of $r_{120}$ and column of $s_{1}$ we have $r_{120} \circ s_{1}$. Once the table is filled, the students can discuss on how to read directly from the table that the composition $\circ$ is not commutative and what the table would look like if it was. Also, it can be discussed how such tables can be used to quickly identify reverse transformations.

\subsubsection{Exploring the Transformations}

Even though the students are now familiar with the concepts, the group $D_{4}$ will pose two new challenges. The first challenge lies within the fact that $D_{4} \nsucceq S_{4}$, as mentioned before. This means that the task of finding all possible transformations can't simply be solved by looking at all the 4 ! positions of vertex numbers. To supply students with an alternative, we refer to the fact that all the elements of $D_{3}$ could be obtained by taking one rotation and one reflection and looking at all possible compositions and that the same is true for $D_{4}$.

We argue that a lot can be learned from clarifying the question why the method of finding all positions of the vertices does not work in the case of the square. For example, we can discuss as to why the map represented in Figure 7 can never be a transformation of the square.

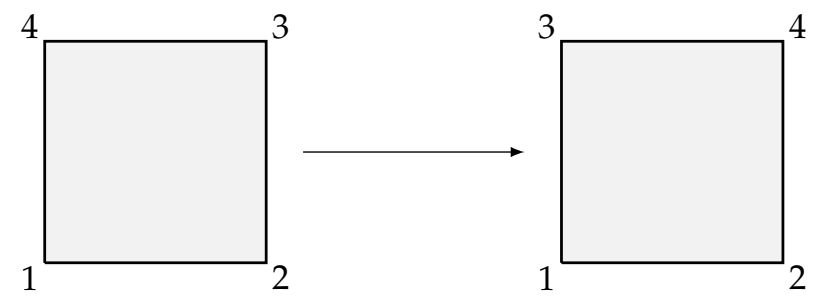

Figure 7. The map above is not a transformation of the square, or, in other words, $(34) \in S_{4} \backslash D_{4}$.

When working with their acrylic glass squares, the students will find out that it is impossible to perform the transformation in the figure above-the positions of 3 and 4 can only be achieved by rotating the square, however, this will also permute 1 and 2 . Thus the students will see that the alternative way presented by the teacher has to be chosen. If, for example, we take $r_{90}$ and $s_{1}$, we get to Table 7 . 
Table 7. Generating the group $D_{4}$ with $r_{90}$ and $s_{1}$.

\begin{tabular}{llllllll}
\hline Transformation & $r_{90}$ & $r_{180}$ & $r_{270}$ & id & $s_{1}$ & $s_{2}$ & $s_{3}$ \\
\hline Construction & $r_{90}$ & $r_{90} \circ r_{90}$ & $r_{90}^{3}$ & $r_{90}^{4}$ & $s_{1}$ & $r_{90} \circ s_{1}$ & $r_{90}^{2} \circ s_{1}$ \\
\hline
\end{tabular}

with the corresponding image (cf. Figure 8).

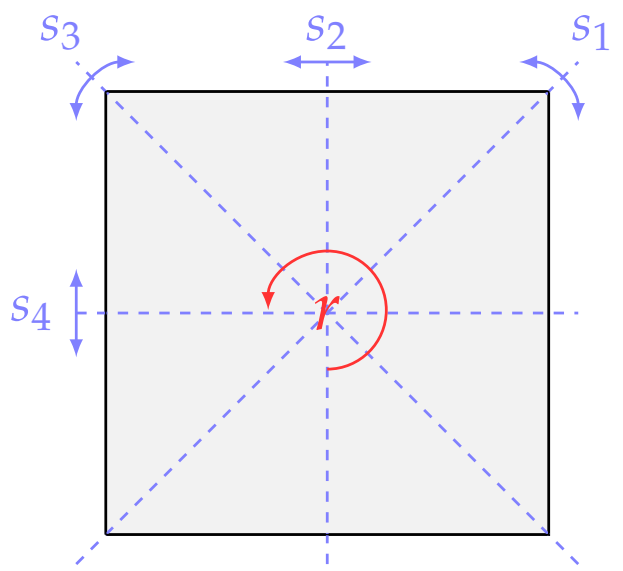

Figure 8. Image containing the elements of $D_{4}$.

The second challenge comes with finding symbols for the reflections. Since the reflections are no longer tied to a specific vertex, a single number can't be assigned to them in a plausible manner. We therefore decided to start with the lower left corner and counting the reflections counter-clockwise as seen in Figure 8, resulting in the set $D_{4}=\left\{\mathrm{id}, r_{90}, r_{180}, r_{270}, s_{1}, s_{2}, s_{3}, s_{4}\right\}$.

\subsubsection{Exploring a Substructure}

After confronting students with similar activities of categorizing and describing all the elements in a table and finding the neutral element as well as all the reverse transformations, we proceed with taking a closer look at the Cayley Table of $D_{4}$. If the first row and column of the tables are arranged in a certain order, one can identify that the rotations build a smaller Cayley Table within the larger one (cf. Table 8).

Table 8. Cayley Table of $D_{4}$.

\begin{tabular}{|c|c|c|c|c|c|c|c|c|}
\hline 0 & id & $r_{90}$ & $r_{180}$ & $r_{270}$ & $s_{1}$ & $s_{2}$ & $s_{3}$ & $s_{4}$ \\
\hline id & id & $r_{90}$ & $r_{180}$ & $r_{270}$ & & & & \\
\hline$r_{90}$ & $r_{90}$ & $r_{180}$ & $r_{270}$ & id & & & & \\
\hline$r_{180}$ & $r_{180}$ & $r_{270}$ & id & $r_{90}$ & & & & \\
\hline$r_{270}$ & $r_{270}$ & $\mathrm{id}$ & $r_{90}$ & $r_{180}$ & & & & \\
\hline$s_{1}$ & & & & & & & & \\
\hline$s_{2}$ & & & & & & & & \\
\hline$s_{3}$ & & & & & & & & \\
\hline$s_{4}$ & & & & & & & & \\
\hline
\end{tabular}

In other words, the rotations build a closed subset where we have the neutral element and reverse transformations. The students can then explore the table to see if they find other subsets with the same properties, obvious candidates are $\{\mathrm{id}\},\left\{\mathrm{id}, s_{1}\right\},\left\{\mathrm{id}, s_{2}\right\},\left\{\mathrm{id}, s_{3}\right\}$ and $\left\{\mathrm{id}, s_{4}\right\}$. It can be discussed that, for example, any subset containing both $r_{90}$ and $s_{1}$ already leads to the entire $D_{4}$ or that the subset of reflections $\left\{s_{1}, s_{2}, s_{3}, s_{4}, \mathrm{id}\right\}$ is not closed under composition. Since in the third teaching unit the group $\left(\left\{\mathrm{id}, r_{90}, r_{180}, r_{270}\right\}, 0\right)$ will reappear in the form of $\mathbb{Z}_{4}$, we will establish the "Rotation Set" $R_{4}=\left\{\mathrm{id}, r_{90}, r_{180}, r_{270}\right\} \subset D_{4}$ to later compare it to $\mathbb{Z}_{4}$. 


\subsection{Unit 3: Introducing the Cyclic Groups $\mathbb{Z}_{n}$}

An introduction of cyclic groups can happen in many different fashions, although usually is done be constructing it via the quotient group $\mathbb{Z} /(n)$. Since $\mathbb{Z}$ is cyclic and thus abelian, every subgroup is normal and one doesn't need to bother with checking whether the construction is well-defined. For dealing with this subject for the first time however, this requires far too many technical tools, so we will adopt the elementary construction by Kirsch [56]. For $\mathbb{Z}_{n}$ he simply defines the operation $\oplus$ on the set $\{0, \ldots, n-1\}$ via

$$
\oplus:(a, b) \mapsto \text { Remainder of } a+b \text { in the division by } n \text {. }
$$

This marks an introduction to cyclic groups solely based on arithmetic of primary school. While still maybe abstract for students, this can be made sense of by referring to mathematical tools they're familiar with.

\subsubsection{Exploring Residue Classes}

Introducing cyclic groups with this approach basically translates to introducing residue classes-Residue classes can be motivated by examples from everyday life:

1. Let's imagine waiting at a station for a train. It is already late, 10:00 p.m. to be precise, and according to the schedule the train will not arrive before 01:00 a.m. A quick mental calculation tells us we need to wait for three more hours, since $10+3=1$.

2. Then we remember that we have to study for a test. Today is 26 November, and the test is precisely in two weeks. Another mental calculation tells us that because November has 30 days, the test will be on 10 December, since $26+14=10$.

Discussing these examples with the students results in two observations: The calculations in the examples are obviously wrong, however, they provide a plausible answer that in the real world makes sense. Thus the question rises whether these weird additions can also be made sense of mathematically. In order to further explore these ideas, we pick up the first example and simplify it: We imagine that one of the moons of Jupiter rotates faster than the earth so that a day there only lasts $5 \mathrm{~h}$. If we lived on this moon, our clocks would therefore only have the 5 digits $0,1,2,3$ and 4 . If it was $4 \mathrm{o}^{\prime}$ clock, then in $3 \mathrm{~h}$ it would be 2 o'clock (cf. Figure 9).

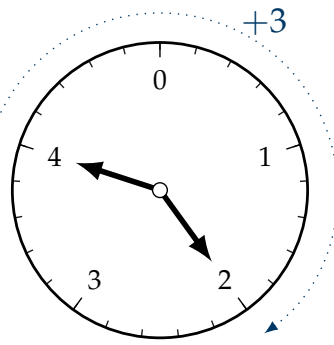

Figure 9. A clock on the fictitious Jupiter moon yielding to $4+3=2$.

A time table on this moon would thus look like presented in Table 9.

Table 9. Timetable on the fictitious Jupiter moon.

\begin{tabular}{llllll}
\hline$\oplus$ & 0 & 1 & 2 & 3 & 4 \\
0 & 0 & 1 & 2 & 3 & 4 \\
1 & 1 & 2 & 3 & 4 & 0 \\
2 & 2 & 3 & 4 & 0 & 1 \\
3 & 3 & 4 & 0 & 1 & 2 \\
4 & 4 & 0 & 1 & 2 & 3 \\
\hline
\end{tabular}

From this we derive that this table looks very similar to the Cayley tables explored in the previous units, evoking the presumption that with similar structures we can also 
make mathematical sense of the given examples. In our concept we have decided to first just look at the residue classes of the natural numbers $\mathbb{N}$ and once the foundations has been laid, expand the concept to $\mathbb{Z}$. Otherwise the minus-symbol gets involved, interfering with the goal that we look at a structure with a single binary operation. Also, negative representatives of the residue classes are not needed at any point in the way described above.

In order to further approach the residue classes, in a next step we will start to explore which numbers are treated as the same in the above mentioned calculations and observe that writing the natural numbers down in a certain way yields a distinctive pattern (cf. Figure 10).

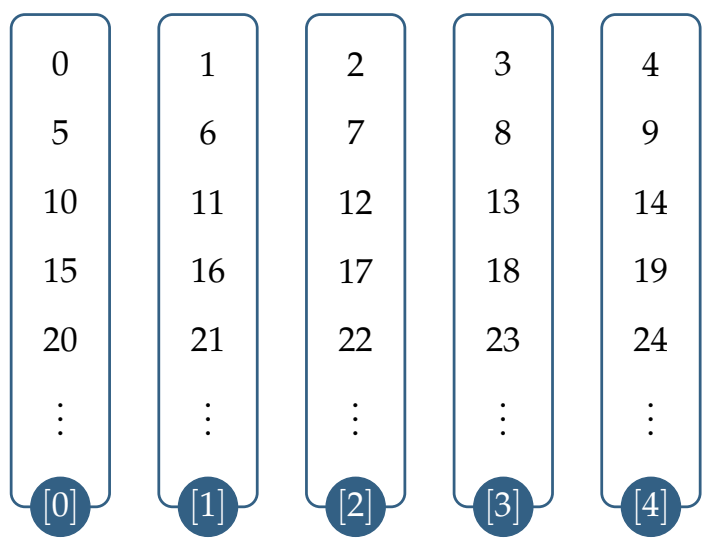

Figure 10. The residue classes visually demonstrated.

Conflating all the numbers in each column to one set gives the sets $[0],[1],[2],[3]$ and [4]. With the students we then can explore that all the numbers in the sets have a gap that is a multiple of 5 and thus we conclude that they all have the same remainder when divided by 5 :

$$
\begin{gathered}
2=0 \cdot 5+2 \\
7=1 \cdot 5+2 \\
12=2 \cdot 5+2 \\
17=3 \cdot 5+2
\end{gathered}
$$

This suggests that these sets are named residue sets with respect to 5 and we can note $R_{5}=\{[0],[1],[2],[3],[4]\}$. For further specification one can also write $R_{5}=\left\{[0]_{5},[1]_{5},[2]_{5}\right.$, $\left.[3]_{5},[4]_{5}\right\}$ when intending to work with other residue classes as well.

\subsubsection{Establishing the Addition of Residue Classes}

With the residue classes established, we can finally start to tackle the examples given at the beginning of the class. For this, we let the students explore different additions. For example, from the equations

$$
\begin{aligned}
8+9 & =17 \\
3+4 & =7 \\
9+13 & =22
\end{aligned}
$$

we can derive that a number in [3] plus a number in [4] always resulted in a number in [2] and we can generalize this observation. Every number $a \in[3]$ can be written as $a=3+k \cdot 5$ and every number $b \in[4]$ can be written as $b=4+\ell \cdot 5$ for some $k, \ell \in \mathbb{N}$, resulting in

$$
a+b=(3+k \cdot 5)+(4+\ell \cdot 5)=2+(k+\ell+1) \cdot 5 \in[2] .
$$


We conclude that we can write [3] $+[4]=[2]$ and view this as an addition of two sets rather than just two numbers. So one entry of the time table on our Jupiter moon is made sense of and the others work exactly the same. It can be further observed that $[0]$ acts as a neutral element and the table of inverse elements looks like Table 10.

Table 10. The inverses in $\mathbb{Z}_{5}$.

\begin{tabular}{cccccc}
\hline $\begin{array}{c}\text { Residue } \\
\text { Class }\end{array}$ & {$[0]$} & {$[1]$} & {$[2]$} & {$[3]$} & {$[4]$} \\
\hline Inverse Class & {$[0]$} & {$[4]$} & {$[3]$} & {$[2]$} & {$[1]$} \\
\hline
\end{tabular}

\subsubsection{Modular Arithmetic}

With the visual ideas of the Cayley table and the Jupiter clock in mind, it is now possible to generalize the findings and also look at multiplication and other residue classes. However, it must be pointed out to students that multiplication does not result in the same structure. For example, in $R_{4}$ the class [2] does not have an inverse class. With this in mind we can now look at equations that look similar to equations on $\mathbb{Z}$ but have an entirely different behaviour. For instance:

- $\quad$ The equation $x^{2}=[0]$ has only one solution when viewed in $\mathbb{Z}$ but two solutions when viewed in $R_{4}$.

- $\quad$ The equation [2] $\odot x=[1]$ has no solution when viewed in $\mathbb{Z}$ but one solution when viewed in $R_{5}$.

The Cayley tables can also be used to solve more advanced equations such as [3] $\cdot(x \oplus$ $[2])=[2]$ in $R_{4}$. Similarly to solving equations in $D_{3}$ and $D_{4}$, the students can either try to make use of the algebraic structure equipped on the sets, or they can simply try out all possible combinations.

\subsubsection{Generalizing the Findings}

With having seen so many different operations and different sets within the three teaching units, we use this for a final take on generalizing the findings, trying to unify the concepts at least to some degree. We do so by putting the Cayley Tables of the rotation subset $R_{4} \subset D_{4}$ and the residue set $R_{4}$ next to each other and slowly manipulate the symbols we invented. By embezzling the brackets, the table of the residue set can be written as Table 11.

Table 11. The Cayley Table of $\mathbb{Z}_{4}$ with slightly changed notation of the elements.

\begin{tabular}{lllll}
\hline$\oplus$ & 0 & 1 & 2 & 3 \\
0 & 0 & 1 & 2 & 3 \\
1 & 1 & 2 & 3 & 0 \\
2 & 2 & 3 & 0 & 1 \\
3 & 3 & 0 & 1 & 2 \\
\hline
\end{tabular}

As for the rotations of the square, we note that it is not necessary to use the measure of the rotation in the symbols. When rotating the square by 90 degrees, each vertex moves exactly one position forward, so one could also write the rotation as $r_{1}$ and change $r_{180} \rightarrow r_{2}$, $r_{270} \rightarrow r_{3}$, and id $\rightarrow r_{0}$ respectively. In the next step, since we're dealing just with rotations anyway, it is no longer necessary to use the letter $r$, the index number sufficiently describes how the square is rotated. So we further simplify the notation to $r_{1} \rightarrow 1, r_{2} \rightarrow 2, r_{3} \rightarrow 3$ and $r_{0} \rightarrow 0$. The Cayley table thus can be written as seen in Figure 11 . 


\begin{tabular}{|c|c|c|c|c|c|c|c|c|c|c|c|c|c|c|c|c|}
\hline o & id & $r_{90}$ & $r_{180}$ & $r_{270}$ & & ० & $r_{0}$ & $r_{1}$ & $r_{2}$ & $r_{3}$ & & o & 0 & 1 & 2 & 3 \\
\hline id & id & $r_{90}$ & $r_{180}$ & $r_{270}$ & & $r_{0}$ & $r_{0}$ & $r_{1}$ & $r_{2}$ & $r_{3}$ & & 0 & 0 & 1 & 2 & 3 \\
\hline$r_{90}$ & $r_{90}$ & $r_{180}$ & $r_{270}$ & id & $\rightsquigarrow$ & $r_{1}$ & $r_{1}$ & $r_{2}$ & $r_{3}$ & $r_{0}$ & $\rightsquigarrow$ & 1 & 1 & 2 & 3 & 0 \\
\hline$r_{180}$ & $r_{180}$ & $r_{270}$ & id & $r_{90}$ & & $r_{2}$ & $r_{2}$ & $r_{3}$ & $r_{0}$ & $r_{1}$ & & 2 & 2 & 3 & 0 & 1 \\
\hline$r_{270}$ & $r_{270}$ & id & $r_{90}$ & $r_{180}$ & & $r_{3}$ & $r_{3}$ & $r_{0}$ & $r_{1}$ & $r_{2}$ & & 3 & 3 & 0 & 1 & 2 \\
\hline
\end{tabular}

Figure 11. Transformation of the Cayley Table of $R_{4}$ when changing notations.

We observe that, apart from the operation symbol, both tables look exactly the samesince the tables encompass the entire information of the structure, this means that rotating squares or calculating sums of residue classes follows the exact same mathematical rules. In other words, the sets we in both cases denoted with $R_{4}$, consist of different symbols but in the context of describing real-life problems the structure of the sets was identical. With the remaining time of the final unit we can coin the name magma for structures equipped with an operation and together with the students search for magmas hidden in the conventional school curriculum, such as $(\mathbb{R},+),(\mathbb{Z},-)$ and so on. Depending on the previous knowledge of the students, we can even illustrate the structure similarity of $(\mathbb{R},+)$ and $\left(\mathbb{R}_{>0}, \cdot\right)$ by using the exponential function (cf. Figure 12$)$.

\begin{tabular}{|c|c|c|c|c|c|c|c|c|}
\hline+ & $\ldots$ & $x$ & . & \multirow{3}{*}{$\rightsquigarrow$} & . & $\cdots$ & $e^{x}$ & \\
\hline : & & : & & & : & & : & \\
\hline$y$ & $\ldots$ & $x+y$ & $\ldots$ & & $e^{y}$ & $\ldots$ & $e^{x+y}$ & $\ldots$ \\
\hline 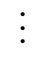 & & : & & & $:$ & & : & \\
\hline
\end{tabular}

Figure 12. Structural similarities of $(\mathbb{R},+)$ and $\left(\mathbb{R}_{>0}, \cdot\right)$ made visible by the exponential function. Both structures just differ in symbolism.

We can conclude the final unit with the observation that many different aspects from the real world look different at the surface, but when describing them mathematically they reveal to be structurally identical. We have explicitly seen this with the examples of rotating polygons and the calculations of times and dates-while different symbols and ways of speaking are used to describe what's going on, on a mathematical level there is absolutely no difference. We can enlighten the students that from this observation an entire mathematical theory emerged and that this theory is finding numerous applications outside of mathematics, namely in computer sciences, physics, chemistry and even music theory.

\section{Conclusions and Outlook}

Even though the contents of abstract algebra may pose challenging hurdles, the rewards can be very beneficial, as pointed out in the first section. Additionally, fostering process-relational skills in mathematics classrooms becomes a more and more relevant issue in education research, and engaging creatively with new mathematical concepts as presented in this course provides an ideal opportunity to train those skills. After all, less reproduction of algorithms is involved, less solving problems to which the solution is already known and just has to be rehashed with slightly different parameters. Mimicking solutions and imitating patterns are no longer strategies that lead to immediate success [40,57], creating the necessity to work with new and creative ways. Abstract algebra emphasizes mathematical understanding in two ways according to Bass [58]: curricular, by making explicit often underdeveloped or overlooked connections among different topics; and cognitive, through inviting new requirements and methods in problem solving activities: "The power of mathematical abstraction is its generality, thus having the potential to conceptually unify many apparently distinct mathematical contexts" ([58], p. 127). Another discussion of the modes of relevance of the term structure in the context of school algebra can be found in [48], describing how problems can be transformed and solved much easier with a structural thinking, recognizing that form and structure should be emphasized in school algebra [59]. 
And on a side note, the most recent mathematical tool in school mathematics is that of differentiation, which by now is over 350 years old. In other words, the thinking in school mathematics is very much a thinking of the past and what mathematics actually encompasses vastly remains hidden throughout the entire school career. Changing this certainly poses many educational problems for learners (cf. [39,44,60,61]), but, as we will see, they can be overcome. An acceptance survey [62] of the Hildesheim Teaching Concept for Abstract Algebra is currently being conducted with individual learners in the laboratory setting - the results of which we will report in a follow-up paper. In the future, however, the concept will be tested empirically, and a transition beyond mere laboratory studies is planned, in order to evaluate the teaching concept in real teaching scenarios at secondary schools in field studies. In the sense of the Design-Based-Research paradigm [63], we will use insights gained from such research to refine the ideas behind the concept as well as revise it both in terms of content and didactical approaches.

Author Contributions: Conceptualization, J.M.V. and P.B.; writing-original draft preparation, J.M.V. and P.B.; writing-review and editing, J.M.V. and P.B. All authors have read and agreed to the published version of the manuscript.

Funding: This research received no external funding.

Institutional Review Board Statement: Not applicable.

Informed Consent Statement: Not applicable.

Data Availability Statement: No new data were created or analysed in this study.

Conflicts of Interest: The authors declare no conflict of interest.

\section{References}

1. Wasserman, N.H. Introducing Algebraic Structures through Solving Equations: Vertical Content Knowledge for K-12 Mathematics Teachers. PRIMUS 2014, 24, 191-214. [CrossRef]

2. McCrory, R.; Floden, R.; Ferrini-Mundy, J.; Reckase, M.D.; Senk, S.L. Knowledge of Algebra for Teaching: A Framework of Knowledge and Practices. J. Res. Math. Educ. 2012, 43, 584-615. [CrossRef]

3. Even, R. The relevance of advanced mathematics studies to expertise in secondary school mathematics teaching: Practitioners views. ZDM Math. Educ. 2011, 43, 941-950. [CrossRef]

4. Veith, J.M.; Bitzenbauer, P. Two Challenging Concepts in Mathematics Education: Subject-Specific Thoughts on the Complex Unit and Angles. Eur. J. Sci. Math. Educ. 2021, 9, 244-251. [CrossRef]

5. Shamash, J.; Barabash, M.; Even, R. From Equations to Structures: Modes of Relevance of Abstract Algebra to School Mathematics as Viewed by Teacher Educators and Teachers. In Connecting Abstract Algebra to Secondary Mathematics, for Secondary Mathematics Teachers; Springer: Cham, Switzerland, 2018; pp. 241-262. [CrossRef]

6. Burn, R. What Are the Fundamental Concepts of Group Theory? Educ. Stud. Math. 1996, 31, 371-377. [CrossRef]

7. Freudenthal, H. What groups mean in mathematics and what they should mean in mathematical education. In Developments in Mathematical Education; Howson, A.G., Ed.; Cambridge University Press: Cambridgeshire, UK, 1973; pp. 101-114. [CrossRef]

8. Wasserman, N.H. Abstract Algebra for Algebra Teaching: Influencing School Mathematics Instruction. Can. J. Sci. Math. Technol. Educ. 2016, 16, 28-47. [CrossRef]

9. Eisenberg, T.A. Begle revisited: Teacher knowledge and student achievement in algebra. J. Res. Math. Educ. 1977, 8, 216-222. [CrossRef]

10. Wasserman, N.H.; Galarza, P. Exploring an Instructional Model for Designing Modules for Secondary Mathematics Teachers in an Abstract Algebra Course. In Connecting Abstract Algebra to Secondary Mathematics, for Secondary Mathematics Teachers; Springer: Cham, Switzerland, 2018; pp. 335-361. [CrossRef]

11. Hunkler, R.F. Achievement of Sixth-Grade Pupils in Modern Mathematics as Related to Their Teachers' Mathematics Preparation. Ph.D. Thesis, The Agricultural and Mechanical College of Texas, College Station, TX, USA, 1968.

12. Hurst, D. The Relationship between Certain Teacher-Related Variables and Student Achievement in Third Grade Arithmetic. Ph.D. Thesis, Oklahoma State University, Stillwater, OK, USA, 1967.

13. Norris, F.R. Pupil Achievement as a Function of an Inservice Training Program on Mathematics Concepts for Sixth Grade Teachers. Ph.D. Thesis, George Peabody College for Teachers, Nashville, TN, USA, 1969.

14. Wasserman, N.H. Exploring Advanced Mathematics Courses and Content for Secondary Mathematics Teachers. In Connecting Abstract Algebra to Secondary Mathematics, for Secondary Mathematics Teachers; Springer: Cham, Switzerland, 2018; pp. 1-15. [CrossRef] 
15. Rouse, W.M. A Study of the Correlation between the Academic Preparation of Teachers of Mathematics and the Mathematics Achievement of Their Students in Kindergarten through Grade Eight. Ph.D. Thesis, Michigan State University, East Lansing, MI, USA, 1967.

16. Copel, W.D.; Doyle, W. Laboratory skill training and student teacher classroom performance. J. Exp. Educ. 1973, 42, 16-21. [CrossRef]

17. Hamann, T. Die "Mengenlehre" im Anfangsunterricht: Historische Darstellung einer Gescheiterten Unterrichtsreform in der Bundesrepublik Deutschland. Ph.D Thesis, University of Hildesheim, Hildesheim, Germany, 2017.

18. Rappaport, D. The New Math and Its Aftermath. Sch. Sci. Math. 1976, 7, 563-570. [CrossRef]

19. Royaumont Seminar. New Thinking in School Mathematics; OECD Publications Center: Paris, France, 1961.

20. OECD. Synopsis für moderne Schulmathematik; Diesterweg: Berlin, Germany, 1966.

21. Kline, M. Why Johnny Can't Add; Random House Inc.: New York, NY, USA 1973.

22. Smith, J.P. Foreshadowing Ideas in Abstract Algebra: Reflections from the Secondary School Mathematics Curriculum Improvement Study. In Connecting Abstract Algebra to Secondary Mathematics, for Secondary Mathematics Teachers; Springer: Cham, Switzerland, 2018; pp. 103-124. [CrossRef]

23. Wilfried, N. Die moderne Algebra und ihre Stellung im heutigen Mathematikunterricht. Didaktik Mathematik 1977, 3, $236-241$.

24. Dubinski, E.; Dautermann, J.; Leron, U.; Zazkis, R. A Reaction To Burn's "What are the Fundamental Concepts of Group Theory?" Educ. Stud. Math. 1997, 34, 249-253. [CrossRef]

25. Liermann, H. Die Behandlung von Restklassengruppen und -ringen im Oberstufenunterricht der Gymnasien. Mathematikunterricht 1966, 2, 95-104.

26. Kropp, G. Der Bildungswert der Mathematik in neuer Sicht und die Bildungsaufgabe des Mathematikunterrichts. Mathematikunterricht 1965, 4, 5-17.

27. Griesel, H. Die Leitlinie Menge-Struktur im gegenwärtigen Mathematikunterricht. Mathematikunterricht 1965, 1, 40-53.

28. Quadling, D.A. The use of the axiomatic method in secondary teaching. Math. Gaz. 1966, 50, 259-275. [CrossRef]

29. Freudenthal, H. Was ist Axiomatik und welchen bildenden Wert kann sie haben? Der Mathematikunterricht 1963, 4, 5-29.

30. Meschkowski, H. Definitionen in der Schulmathematik. Der Mathematikunterricht 1965, 4, 94-108.

31. Servais, W.; Varga, T. Treatment of the Axiomatic Method in Class. In Teaching School Mathematics; Penguin Books: London, UK, 1971; pp. 124-150.

32. Howson, A.G. The Role of axioms in contemporary mathematics and in mathematical education. In Developments in Mathematical Education; Cambridge University Press: Cambridgeshire, UK, 1973; pp. 262-271.

33. Steiner, H. Menge, Struktur, Abbildung als Leitbegriffe für den modernen mathematischen Unterricht. Mathematikunterricht 1965, 1, 5-19.

34. Steiner, H. Einfache Verknüpfungsgebilde als Vorfeld der Gruppentheorie. Mathematikunterricht 1966, 2, 5-18.

35. Dieudonne, J. Vortrag auf der 27. Tagung zur Pflege des Zusammenhangs von Universität und Höherer Schule in Münster. Didaktik Mathematik 1962, 4, 171-183.

36. Leppig, M. Beispiele zum Rechnen in endlichen Gruppen. Mathematikunterricht 1966, 2, 39-49.

37. Kirsch, A. Über die enaktive Repräsentation von Abbildungen, insbesondere Permutationen. Didaktik Mathematik 1965, 3, 169-194.

38. Wasserman, N.H. Connecting Abstract Algebra to Secondary Mathematics, for Secondary Mathematics Teachers; Springer: Cham, Switzerland, 2018.

39. Selden, A.; Selden, J. Errors and misconceptions in college level theorem proving. Proc. Second. Int. Semin. Misconceptions Educ. Strateg. Sci. Math. 1987, 3, 457-470.

40. Weber, K.; Larsen, S. Teaching and Learning Group Theory. In Making The Connection; Carlson, M.P., Rasmussen, C., Eds.; Mathematical Association of America: Washington, DC, USA, 2008; pp. 139-151.

41. Zbiek, R.M.; Heid, M.K. Making Connections from the Secondary Classroom to the Abstract Algebra Course: A Mathematical Activity Approach. In Connecting Abstract Algebra to Secondary Mathematics, for Secondary Mathematics Teachers; Springer: Cham, Switzerland, 2018; pp. 189-209. [CrossRef]

42. Baldinger, E.E. Learning Mathematical Practices to Connect Abstract Algebra to High School Algebra. In Connecting Abstract Algebra to Secondary Mathematics, for Secondary Mathematics Teachers; Springer: Cham, Switzerland, 2018; pp. 211-239. [CrossRef]

43. Suominen, A.L. Abstract Algebra and Secondary School Mathematics Connections as Discussed by Mathematicians and Mathematics Educators. In Connecting Abstract Algebra to Secondary Mathematics, for Secondary Mathematics Teachers; Springer: Cham, Switzerland, 2018; pp. 149-173. [CrossRef]

44. Melhuish, K.; Fagan, J. Connecting the Group Theory Concept Assessment to Core Concepts at the Secondary Level. In Connecting Abstract Algebra to Secondary Mathematics, for Secondary Mathematics Teachers; Springer: Cham, Switzerland, 2018 ; pp. 19-45. [CrossRef]

45. Wasserman, N.H. Making Sense of Abstract Algebra: Exploring Secondary Teachers' Understandings of Inverse Functions in Relation to Its Group Structure. Math. Think. Learn. 2017, 19, 181-201. [CrossRef]

46. Brown, A.; DeVries, D.J.; Dubinskiy, E.; Thomas, K. Learning Binary Operations, Groups, and Subgroups. J. Math. Behav. 1997, 16, 187-239. [CrossRef]

47. Larsen, S. Making Sense of Abstract Algebra: Reinventing the concepts of group and isomorphism: The case of Jessica and Sandra. J. Math. Behav. 2009, 28, 119-137. [CrossRef] 
48. Lee, Y.; Heid, M.K. Developing a Structural Perspective and Its Role in Connecting School Algebra and Abstract Algebra: A Factorization Example. In Connecting Abstract Algebra to Secondary Mathematics, for Secondary Mathematics Teachers; Springer: Cham, Switzerland, 2018; pp. 291-318. [CrossRef]

49. Van den Heuvel-Panhuizen, M.; Drijvers, P. Connecting the Group Theory Concept Assessment to Core Concepts at the Secondary Level. In Encyclopedia of Mathematics Education; Springer: Cham, Switzerland, 2020. [CrossRef]

50. Cook, J.P. Monster-Barring as a Catalyst for Bridging Secondary Algebra to Abstract Algebra. In Connecting Abstract Algebra to Secondary Mathematics, for Secondary Mathematics Teachers; Springer: Cham, Switzerland, 2018; pp. 47-70. [CrossRef]

51. Blanton, M.L.; Stephens, A.; Knuth, E.; Gardiner, A.M.; Isler-Baykal, I.; Kim, J.-S. The Development of Children's Algebraic Thinking: The Impact of a Comprehensive Early Algebra Intervention in Third Grade. J. Res. Math. Educ. 2015, 46, 39-87. [CrossRef]

52. Kaput, J. What is algebra? What is algebraic reasoning? In Algebra in the Early Grades; Kaput, J., Carraher, D.W., Blanton, M.L., Eds.; Routledge: New York, NY, USA, 2008; pp. 5-17

53. Carraher, D.W.; Martinez, M.V.; Schliemann, A.D. Early algebra and mathematical generalization. ZDM Math. Educ. 2007, 40, 3-22. [CrossRef]

54. Papic, M. An Early Mathematical Patterning Assessment: Identifying young Australian Indigenous children's patterning skills. Math. Educ. Res. J. 2015, 27, 519-534. [CrossRef]

55. Pincheira, N.; Alsina, A. Teachers' Mathematics Knowledge for Teaching Early Algebra: A Systematic Review from the MKT Perspective. Mathematics 2021, 9, 2590. [CrossRef]

56. Kirsch, A. Elementare Zahlbereichserweiterungen und der Gruppenbegriff. Der Mathematikunterricht 1966, 2, 50-66.

57. Dubinskiy, E.; Dautermann, J.; Leron, U.; Zazkis, R. On learning fundamental concepts of group theory. Educ. Stud. Math. 1994, 27, 267-305. [CrossRef]

58. Hyman, B. Understanding School Mathematics in Terms of Linear Measure and Discrete Real Additive Groups. In Connecting Abstract Algebra to Secondary Mathematics, for Secondary Mathematics Teachers; Springer: Cham, Switzerland, 2018; pp. 125-147. [CrossRef]

59. Alvarez, J.M.; White, D. Making Mathematical Connections Between Abstract Algebra and Secondary Mathematics Explicit: Implications for Curriculum, Research, and Faculty Professional Development. In Connecting Abstract Algebra to Secondary Mathematics, for Secondary Mathematics Teachers; Springer: Cham, Switzerland, 2018; pp. 175-185. [CrossRef]

60. Ticknor, C.S. Situated learning in an abstract algebra classroom. Educ. Stud. Math. 2012, 81, 307-323. [CrossRef]

61. Hart, E. Analysis of the proof-writing performance of expert and novice students in elementary group theory. In Research Issues in Undergraduate Mathematics Learning: Preliminary Analyses and Reports; Mathematical Association of America: Washington, DC, USA, 1994; pp. 49-62.

62. Jung, W. Probing acceptance: A technique for investigating learning difficulties. In Research in Physics Learning. Theoretical Issues and Empirical Studies; Duit, R., Goldberg, F., Niedderer, H., Eds.; IPN: Kiel, Germany, 1992; pp. $278-295$.

63. Anderson, T.; Shattuck, J. Design-Based Research: A Decade of Progress in Education Research? Educ. Res. 2012, 41, 16-25. [CrossRef] 